\title{
A project design of virtual cloud desktop system based on OpenStack
}

\author{
Zhi-hui Shang ${ }^{1, a}$, Jian-wei Zhang ${ }^{2, b, \ddagger}$, Zeng-yu Cai ${ }^{1}$, Lin-lin Ma ${ }^{1}$, Chen Yuan ${ }^{1}$, \\ Chun-hui $\mathrm{Hu}^{2}$ \\ ${ }^{1}$ School of Computer and Communication Engineering, Zhengzhou University of Light Industry, \\ Zhengzhou 450002, China \\ ${ }^{2}$ Software Engineering College, Zhengzhou University of Light Industry, Zhengzhou 450002, China \\ aemail:1126483520@qq.com \\ bemail:2453651858@qq.com
}

Keywords: Cloud computing, virtual cloud desktop, OpenStack, dynamic migration, scheduling strategy, A pool of virtualized resources.

Abstract. In this paper, a virtual cloud desktop system is built in the open source cloud platform. And a design project of virtual cloud desktop system based on OpenStack is proposed through the analysis of the overall scheme of the system. First, this paper made a detailed description for the framework of the overall design project of virtual cloud desktop system based on OpenStack and then we introduced the work flow of the virtual cloud desktop system and made a deep analysis for every function module in the process. Finally, this paper made a detailed analysis of the key technology of this system. The design project of virtual cloud desktop system proposed in this paper has great significance for the intelligent virtualization of the laboratory and an important reference value for the building of virtual cloud desktop system.

\section{Introduction}

With the rapid development of virtualization and cloud computing[1], the traditional laboratory was replaced by virtual cloud laboratory gradually[2]. The server of traditional desktop virtualization technology is usually a cluster which is composed of the virtualization technology of server making use of multiple servers. And the server physical host with strong performance virtualized several virtual machine instances. Then, the cluster composed by these virtual machine instances is regarded as a virtual resources pool providing the service of virtualization of desktop or other services.

But, the virtual cloud desktop resources pool built in a traditional way has not realized the load balancing. Since OpenStack supports to build cloud platform, which itself is open and open-source, so OpenStack is a free software and open source project aiming at providing software for the construction and management of the public and the private cloud[3,4]. The design and source code of the module is completely open, which contributes to the scalability of OpenStack and so developers can conveniently add new functions and modules to the original one[5].

In this paper, OpenStack platform is used as the desktop virtualization server for improving the framework of OpenStack. By doing this, the whole system would be more suitable to the desk virtualization system. At the same time, as the virtual desktop cloud server, it also offers the user the manager module for better control of the virtual desktop cloud system. Because the default scheduling module of OpenStack is based on random algorithms and there is no loading and scheduling for the dynamic of virtual cloud platform, so server of system also provides pre-scheduling modules for finishing the work. The whole virtual desktop delivery process of the user can be realized by all modules provided by the server.

\section{The whole design project of virtual cloud desktop based on OpenStack}

\section{The architecture design of system}

This paper aims to the design of the desktop virtualization and to build it by means of the opensource cloud computing infrastructure platforms of OpenStack. However, there are four levels on the 
virtual cloud platform from the top to the bottom: the hardware layer, the virtual layer, the scheduling layer and the terminal access layer. The construction figure of cloud platform is shown in Fig. 1.

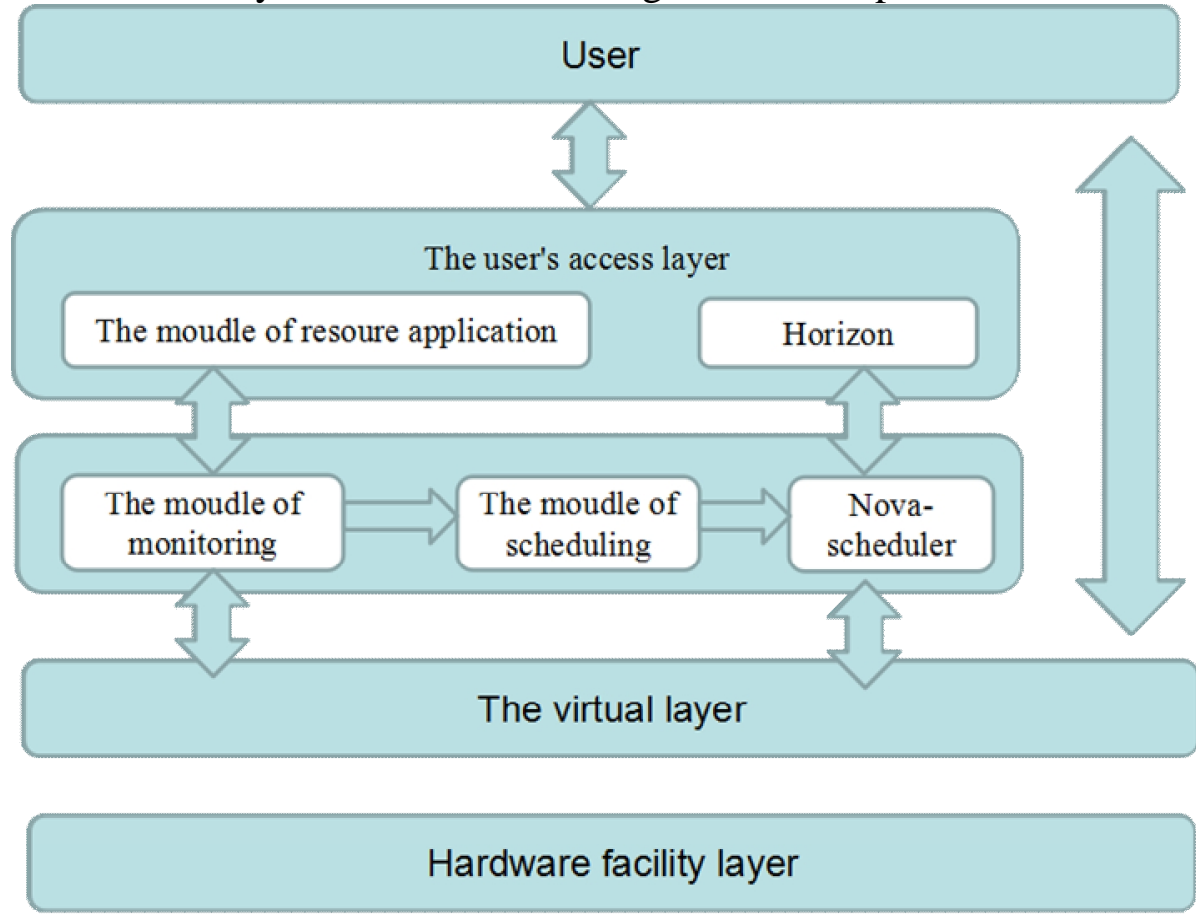

Fig. 1 The layered architecture of cloud system

However, OpenStack only provides the basic platform of cloud computing, that is IaaS. In this paper, on the one hand, the building of virtual desktop resource pool needs the Nova and Horizon project of OpenStack .on the other hand, the Nova module and Horizon module should be managed through the other modules designed by this system. And to provide friendlier virtual desktop delivery process for the user[6]. OpenStack has provided many ready-made tools or called modules for servers for realizing the desktop virtualization. For example, in this paper, Computer (Nova) and image Management (Glance) these two project modules and other necessary modules of OpenStack have been used in the construction of desktop virtualization. These modules are mainly the service framework of OpenStack.

\section{The design of system module}

In this paper, first, OpenStack platform is used for building a reliable IaaS platform on the basis of a operational system Ubuntu Server. The modules of OpenStack chosen by developmental platform proposed in this paper conclude: OpenStack Compute (Nova), OpenStack, Object Storage (Swift), OpenStack Image Service (Glance), Identity (KeyStone) and DashBoard (Horizon)[7]. Nova is used for the management of the lifecycle of virtual cloud terminal instance in OpenStack environment and its responsibilities include the building of virtual desktop, scheduling and destruction, etc. Swift provides an object storage system of high availability and scalability. Glance is a virtual mirror image storage, query and retrieval system. Keystone is responsible for the functions of authentication, service rule and service permission token. Horizon provides a convenient web management interface.

The development of virtual cloud desktop is mainly divided into three modules: the module of load monitoring, the module of virtual desktop scheduling, the module of resource management. And the three module of system are realized through four parts:

1) Virtual desktop manager. It managers the virtual desktop of the user. When users log into the system and are authenticated, they can operate their own virtual desktop. When users log into the system through a virtual desktop client, they can see all the virtual desktop that they have and then they can operate their own virtual desktop. Such as restart, delete, pause and resume operations, etc.

2) User manager. The main function of user manager is to manager all the users in the whole virtual desktop cloud system, which provides services of login and register for the users. The users can 
get the permission through this manager when they log in and if users want to interact directly with OpenStack cluster later, they can be authenticated through the permission that was gained just.

3) The pre-scheduler. Because there are a lot of computing nodes in OpenStack cluster and if scheduling through the Nova-schedule module provided by OpenStack, the time of getting the result of scheduling would be long when the requests and loading are excessive. However, the pre-scheduler collect the loading condition of every OpenStack cluster managed by managers and is responsible for updating the list of load-magnitude. So, when there is a new request, it can be dispatched into a smaller OpenStack cluster quickly by the pre-scheduler.

4) Call agent. The agent module is the bridge of the desktop cloud manager interacting with the outside world [8]. The user of virtual desktop can be linked to the manager. At the same time, the manager can manage every OpenStack and initiate API call through this call agent $[9,10]$, In addition, there is another function of this call agent. It can send the call information to every OpenStack cluster at intervals for confirming whether the OpenStack cluster is being managed. The reply of OpenStack cluster to the call information is the message queue of the cluster, computing nodes, management node and the usage of CPU, etc. Al these information is sent to the pre-scheduling module for using. The basic flow diagram of virtual cloud desktop system is shown in Fig. 2.

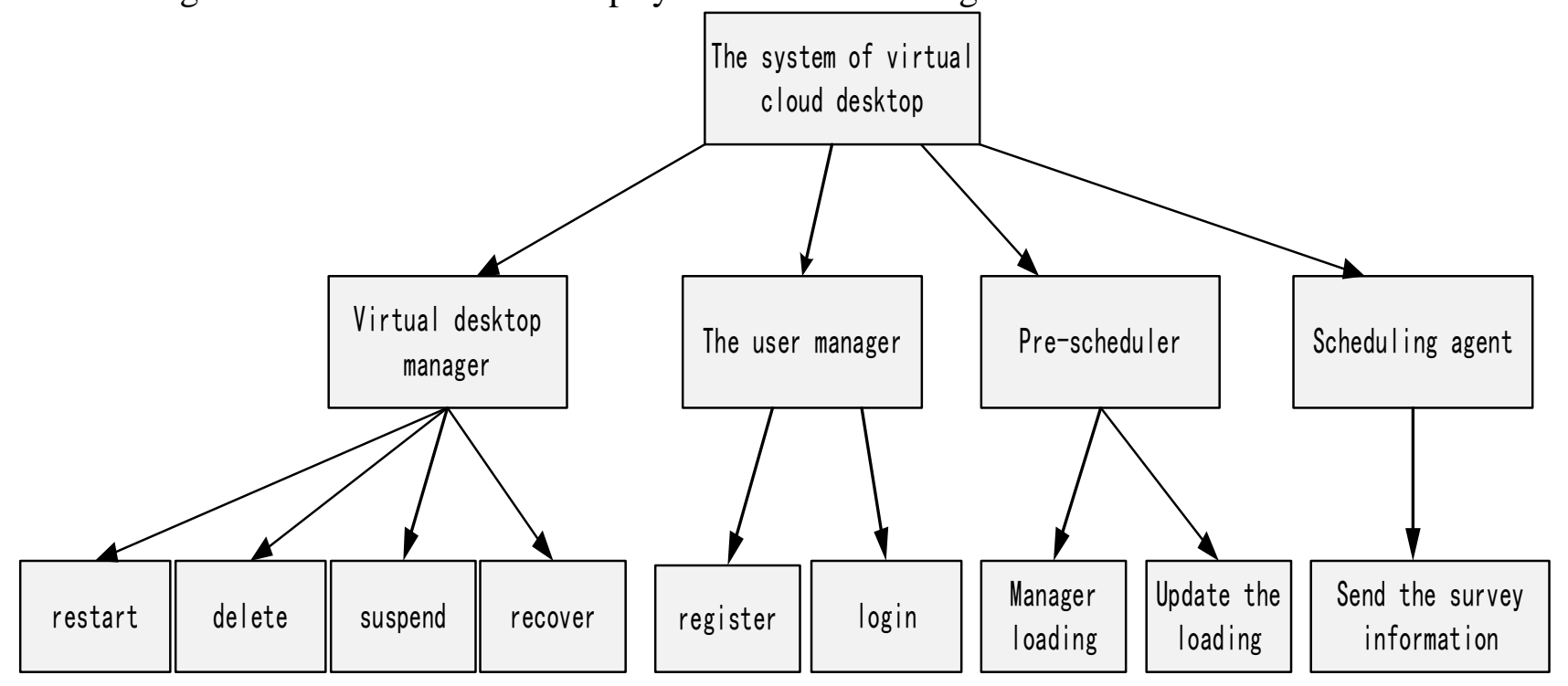

Fig. 2 The basic flow diagram of virtual cloud desktop system

\section{The work flow of virtual cloud desk based on OpenStack technology}

The system mainly researches on resource scheduling of virtual cloud platform including initialization assign of virtual machine and the resource scheduling at the time of dynamic migration and the destruction of virtual cloud desktop. And it also manages the resource's life cycle of virtual cloud desktop. The scenario of scheduling system platform of virtual cloud desktop shows in Fig. 3. 


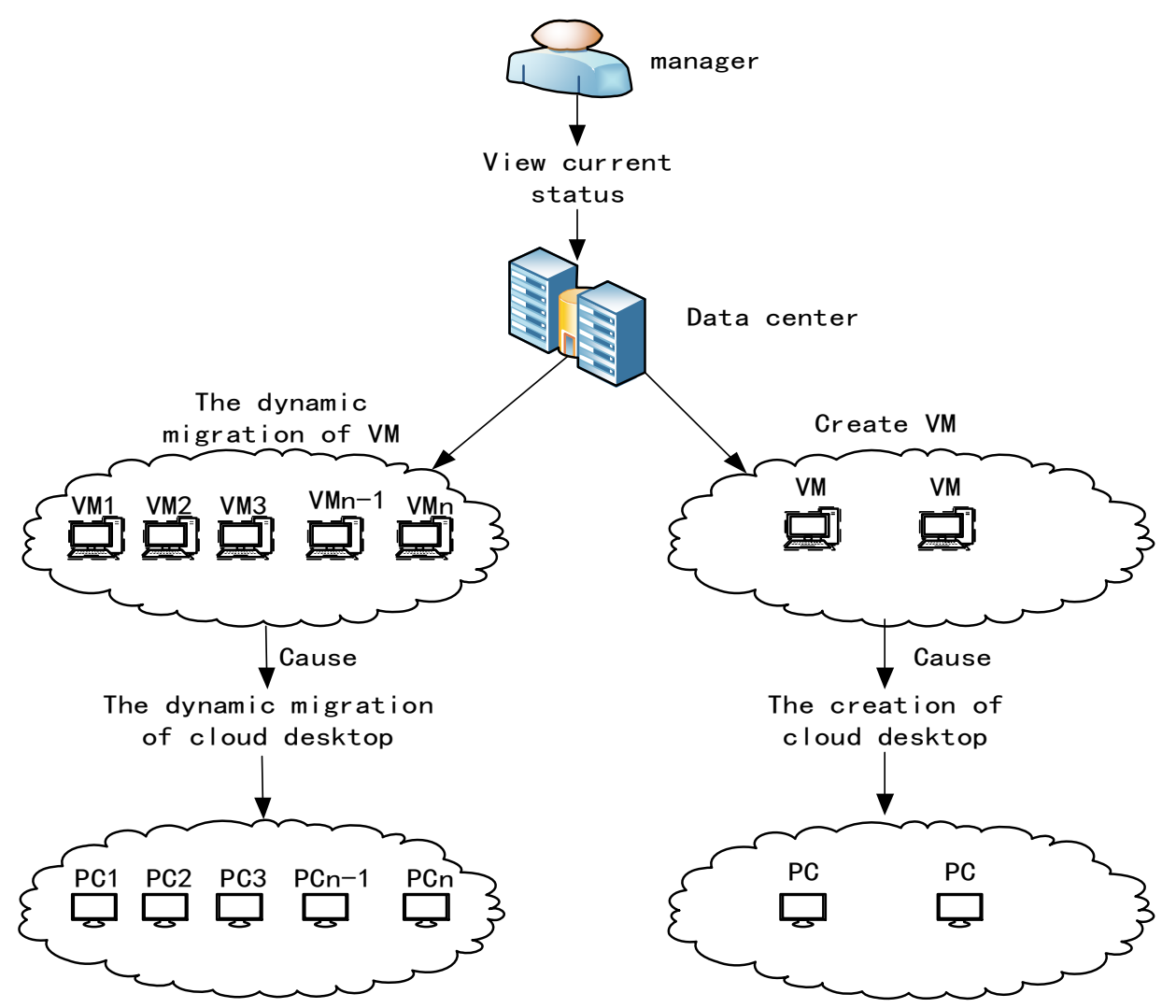

Fig. 3 The scenario of scheduling system platform

1) The request of administrator: the administrator initiates the requests of checking equipment and managing equipment through logging in the web interface.

2) The management of resource status: administrator by means of identity feature requests to check the business (the resource status of virtual machine or physical machine), and submits requests the data center. Then the data center schedules resource monitoring module and performs the dynamic migration operation. Apart from the manual call of the administrator, resource status monitoring module can also be performed at intervals. The operation of resources status monitoring can also be performed automatically in the background, which would leads to automatic triggering and automatic migration. Administrator also can create virtual cloud desktop through this system.

3) Feedback: the resources status module feedback the current resource information to the data center in real time.

4) Log information: after the dynamic migration of virtual machine, the relevant migrant records will be fed back to the log information of database.

For the above scenarios, the virtual cloud desktop platform based on OpenStack designed and realized in this paper are capable of managing the system of data center uniformly and can change the creation and dynamic migration of virtual cloud desktop through the dynamic scheduling migration of virtual machine.

\section{The analysis of key technologies}

Virtual cloud desktop system based on OpenStack can generate a virtual desktop instance through three strategies:

1) A virtual desktop instance is generated in advance by using pre-loading strategy. But there are two problems of this strategy: First, the problem of choosing the time node. Second, the conflicts of IP address.

2) A virtual desktop instance is generated by using the Snapshot strategy. But there are two problems of this strategy: First, time of looking for file by snapshot is too long, and it will 
decrease the performance of virtual machine. Second, to restore the virtual desktop will cause the waste of original image network resources.

3) Use of mixed strategy, which is the combination of pre-loading and restored snapshot.

The cloud environment not only provides experimental environment for teaching, but also cloud servers for teachers. If the virtual desktop snapshot using by all teaching and learning environment is created in advance, it will cause unnecessary consumption of resources. So, the creation of snapshot and the restore of snapshot can be completed before the start time $t 1$ of this course. After the completion of use, the virtual desktop instances based on snapshot will be destructed in order to release system resources maximum.

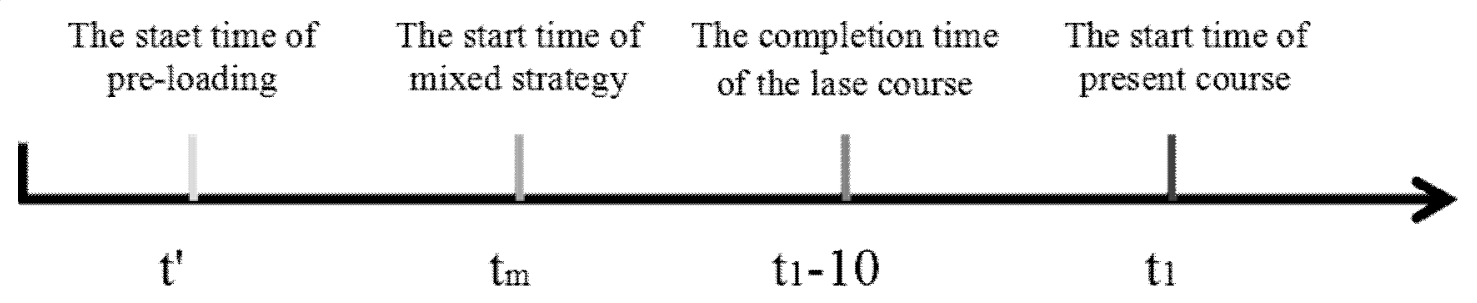

Fig. 4 The timeline of mixed optimized strategy of virtual desktop

Therefore, this project proposed virtual desktop scheduling techniques based on pre-loading and the restore of snapshots by combining pre-loading with the snapshot restore strategy. Just as showed in Fig. 4 , selecting the appropriate pre-loading time automatically before time $\mathrm{t} 1$ and creating bulk experimental teaching virtual desktop environment by restoring the snapshot, which is needed by time t1 and not influencing the normal teaching task. it can create virtual desktop environment and can lower the loading of cloud environment in a great degree.

System uses hybrid cross-scheduling strategy and strategy will have different effects when the operational number of scheduling is different. The scheduling strategy will be optimized when the operational number is 30 . Compared with the two former strategies, cross- scheduling has the obvious convergence effect on the scheduling number of virtual task resource and the optimal path.

\section{Conclusions}

The system build the operational resource pool of virtual cloud platform through a lot of OpenStack clusters then manage these OpenStack clusters by virtual cloud desktop management. And the pre-scheduling module is added in OpenStack clusters. This platform can effectively consolidate server resources and provide resource service of virtual cloud platform for users on campus, so that the application system can get the independent running environment. Although the application of OpenStack in virtual cloud is still at an early stage, with the development of technology and declining costs, OpenStack technology will be fully applied in the virtual cloud scheduling in the near future. The key technology discussed in this paper is a matter of scheduling strategy that must be faced with. How to design a practical scheduling algorithm and establish an effective virtual cloud management platform is our next focus of study.

\section{Acknowledgements}

This work is financially supported by 2016 Outstanding Innovation Science and Technology Youth Project of the Innovation Scientists and Technicians Plan of Henan Province (164100510019). Zhengzhou science and technology key project(153PKJGG26). Science and technology innovation fund project for graduates of Zhengzhou University of Light Industry.

\section{References}

[1] Zhang Q, Cheng L, Boutaba R.Journal of internet services and applications, Vol.1(2010), p.7-18. 
[2] Jian Wei Zhang, Zhi Hui Shang, Chen Yuan, et al. Research and Progress on Virtual Cloud Laboratory[C]. MATEC Web of Conferences: EDP Sciences,2016:01041,p1-6.

[3]Corradi A,Fanelli M, Foschini L. Future Generation Computer Systems, Vol.32(2014),p .118-127.

[4] Leander Beernaert, Miguel Matos, Ricardo Vilaça, Rui Oliveira.Secure and Dependable Middleware for Cloud Monitoring and Management, 2012-12.

[5] Yi Zhang. Design and Implementation of The Virtual Desktop Cloud Server-end based on OpenStack .South China University of Technology, In Chinese.2013.

[6] Bo Fu. Research of the Cloud Computing Platform and Scheduling Scheme Based on OpenStack. Beijing Jiaotong University, In Chinese. 2014.

[7] Lili Zhang. The Key Technology Research of Virtual Maching Resource Scheduling Based on OpenStack.Beijing Jiaotong University, In Chinese. 2012.

[8] Zhao W, Wang Z. Acm Sigops Operating Systems Review, Vol.43 ,(2009), p.37-47.

[9] Xianfeng Sheng, Junchuan Ji, Xiaojun Zhou, et al.Private Cloud APCS Platform Design Based on Virtualization Technology. Computer Engineering, In Chinese. Vol.8(2012), p.210-212.

[10] Shaoka Zhao, Liyao LI, Xiao Ling, et al. Architecture and scheduling scheme design of Tsinghua Cloud based on OpenStack. Journal of Computer Applications, In Chinese.Vol.12(2013), p.3335-3338.

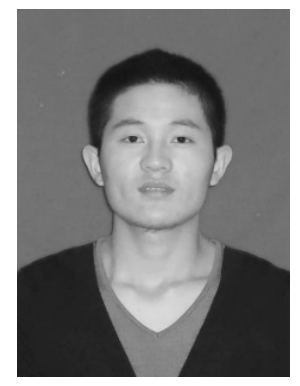

Zhi-hui SHANG, born in November, 1989, Henan, grades: A graduate student at Zhengzhou University of Light Industry, Scientific interest: Cloud computing and computer network .

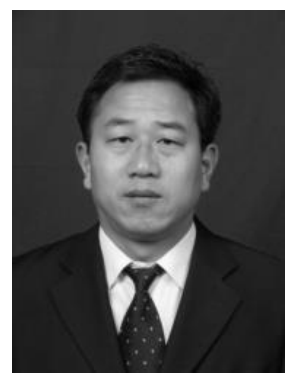

†Corresponding author

Jian-wei ZHANG, born in April, 1971, Henan, P R china Current position, grades: Professor at Zhengzhou University of Light Industry, China University studies: $\mathrm{PhD}$ degree from The PLA Information Engineering University in China Scientific interest: broadband information network and network security . 